\title{
Emerging Concept in DNA Methylation: Role of Transcription Factors in Shaping DNA Methylation Patterns
}

\author{
CLAIRE MARCHAL AND BENOIT MIOTTO* \\ Université Paris Diderot, Sorbonne Paris Cité, Epigenetics and Cell Fate, Paris, France
}

\begin{abstract}
DNA methylation in mammals is a key epigenetic modification essential to normal genome regulation and development. DNA methylation patterns are established during early embryonic development, and subsequently maintained during cell divisions. Yet, discrete site-specific de novo DNA methylation or DNA demethylation events play a fundamental role in a number of physiological and pathological contexts, leading to critical changes in the transcriptional status of genes such as differentiation, tumor suppressor or imprinted genes. How the DNA methylation machinery targets specific regions of the genome during early embryogenesis and in adult tissues remains poorly understood. Here, we report advances being made in the field with a particular emphasis on the implication of transcription factors in establishing and in editing DNA methylation profiles.
\end{abstract}

J. Cell. Physiol. 230: 743-75I, 20I5. (c) 2014 The Authors. Journal of Cellular Physiology published by Wiley Periodicals, Inc.

DNA methylation is a well-studied epigenetic modification in mammalian genomes, discovered in 1948. It is involved in a number of essential cellular processes such as transcription regulation, cellular differentiation, cellular identity maintenance, $X$ inactivation, gene imprinting, and the cellular response to environmental changes (Klose and Bird, 2006; Guibert and Weber, 20I3; Smith and Meissner, 20I3; Subramaniam et al., 2014). DNA methylation has proved to be a dynamic process, requiring continuous regulation and potentially having an important regulatory role for tissuespecific differentiation or cellular signaling. Indeed, the analysis of the distribution of DNA methylation at the genome scale, and nowadays at the single-base resolution, in different physiological and pathological states, unraveled that local changes in DNA methylation contribute to cell-type specific variation in gene expression. Furthermore, aberrant DNA methylation patterns are documented in a number of human diseases from Immunodeficiency, Centromere instability, and Facial anomalies (ICF) syndrome to cancer, and contribute to the onset or development of these diseases (Smith and Meissner, 2013; Weng et al., 2013; Subramaniam et al., 20l4). Needless to say, these discoveries also fuel the promising idea that therapeutic strategies targeting DNA methylation can be used in the prevention and the treatment of cancer and other human diseases, including neuro-developmental disorders (Weng et al., 20I3; Subramaniam et al., 2014). As an example, antipsychotic drugs clozapine and sulpiride, combined with histone deacetylase inhibitor valproate, have a beneficial action in schizophrenia and bipolar patients, maybe because they revert the aberrant DNA methylation status at GABAergic gene promoters (Dong et al., 2008). In 2004, 5-azacytidine (Vidaza $^{\text {TM }}$, Celgene Corporation, Summit, NJ). A drug blocking DNA methylation, received approval by the Food and Drug Administration for the treatment of myelodysplastic syndromes (Kaminskas et al., 2005).

In somatic cells, DNA methylation mostly occurs in the CpG context, and $70-80 \%$ of $C_{p}$ Gs are methylated in mammalian genomes (Lister et al., 2009; Guibert and Weber, 2013; Ziller et al., 20I3). DNA methylation is present in gene bodies, intergenic regions, DNA repeats and endogenous retrotransposons. On the contrary, $C_{p G}$-rich regions of the genome, called CpG islands, are protected from DNA methylation and, conversely, they coincide with promoters, replication origins and cis-regulatory transcriptional elements (Lister et al., 2009; Ziller et al., 2013). DNA methylation patterns established during embryonic development are faithfully copied through somatic cell divisions. The ubiquitously expressed DNA methyltransferase DNMTI is predominantly responsible for copying and maintaining DNA methylation pattern after DNA replication, while the de novo DNA methyltransferases DNMT3A and DNMT3B are involved in the establishment of new DNA methylation patterns (Fig. I) (Okano et al., 1999; Sharif et al., 2007; Subramaniam et al., 2014). While mostly stable it has been soon noticed that in certain developmental processes and human diseases DNA methylation can be, globally or locally, erased (Fig. I). Two main demethylation pathways have been described over the years. Methyl-CpG can be passively removed by blocking methylation of newly synthesized DNA during DNA replication. The methyl-group can also be enzymatically oxidized by the TET (ten-eleven translocation

This is an open access article under the terms of the Creative Commons Attribution-NonCommercial-NoDerivatives License, which permits use and distribution in any medium, provided the original work is properly cited, the use is non-commercial and no modifications or adaptations are made.

$\mathrm{BM}$ and $\mathrm{CM}$ wrote the manuscript.

Contract grant sponsor: Ligue contre le Cancer (Comité de Paris) and Marie Curie International Re-integration;

Contract grant number: PIRG07-GA-2010-268448.

*Correspondence to: Benoit Miotto, CNRS, UMR 7216,

Epigenetics and Cell Fate, University Paris Diderot, Bât Lamarck, 35 Rue Hélène Brion, 75013 Paris, France.

E-mail benoit.miotto@univ-paris-diderot.fr

Manuscript Received: 5 September 2014

Manuscript Accepted: I October 2014

Accepted manuscript online in Wiley Online Library (wileyonlinelibrary.com): 6 October 2014.

DOI: $10.1002 /$ jcp.24836 


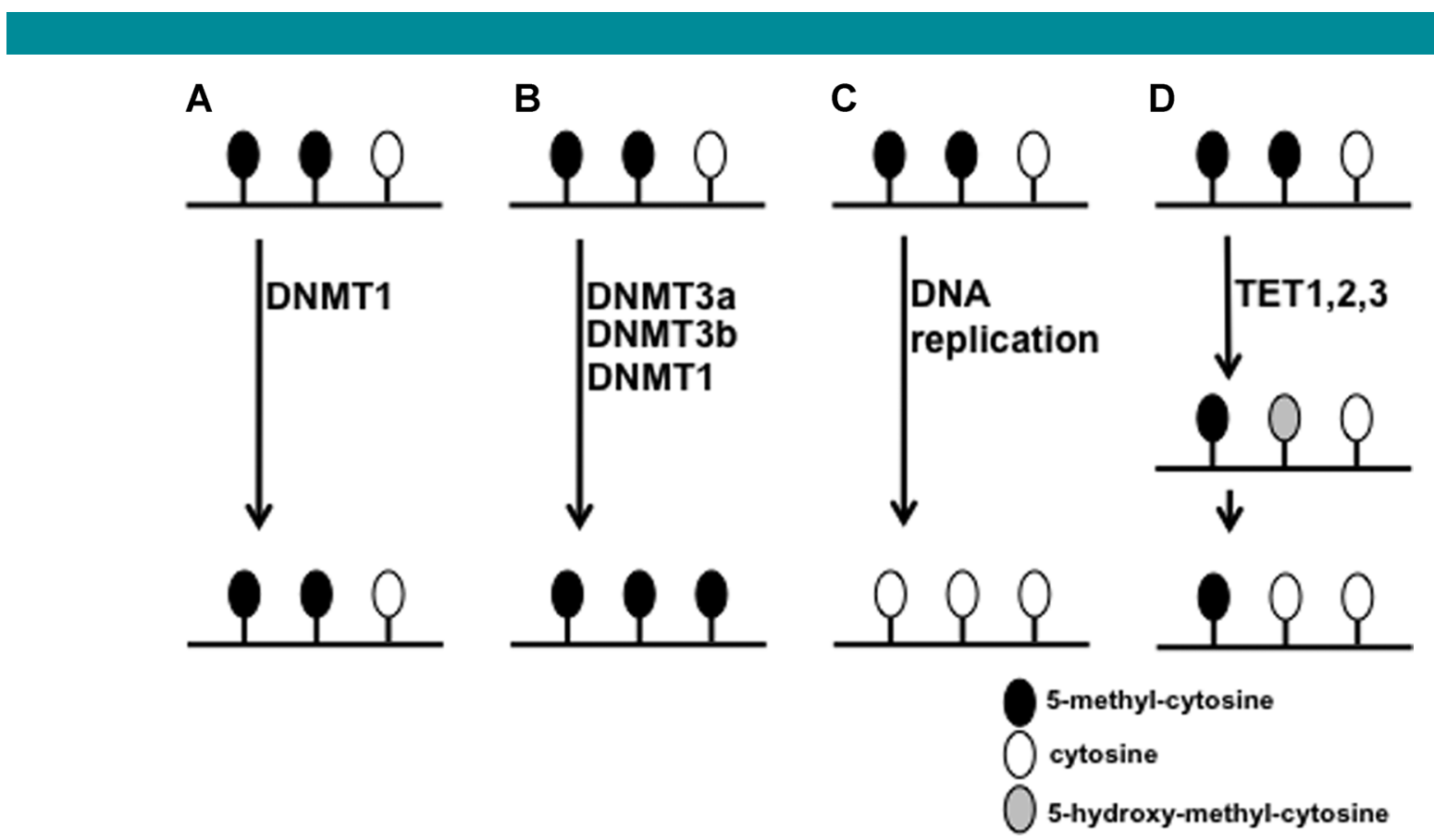

Figure I. Overview of the DNA methylation and demethylation pathway. (A) DNMTI is responsible for the maintenance of DNA methylation during DNA replication. It recognizes hemi-methylated CpG, thanks to its interaction with co-factor UHRFI, and it adds methylation on the un-methylated strand. Black bubbles: methylated CpG. Empty bubbles: un-methylated CpG. (B) DNMT3A/B are responsible for de novo DNA methylation. They establish new patterns of methylation directly from unmethylated CpG-containing sequences. In the embryo, their activity is modulated by a catalytically inactive family member DNMT3L. (C) Passive demethylation occurs through loss of DNMTI/3 activity in actively dividing cells. Loss can be attributed to post-translational modifications, gene mutations, gene silencing or any other mechanism that will eventually lead to DNMT activity inhibition. (D) Active DNA demethylation is catalyzed by the TET family of enzymes. TETI, 2 and 3 can oxydate $5 \mathrm{mC}$ into $5 \mathrm{hmC}$ (represented in grey bubbles), and eventually oxidate $5 \mathrm{hm}$ C into 5 -formylcytosine and 5-carboxy-cytosine. None of these bases is recognized by DNMTs causing loss of DNA methylation during DNA replication. In addition, these oxidated bases are recognized by the base-excision repair (BER) pathway and catalytically removed.

methylcytosine dioxygenase) family enzymes to give rise to 5-hydroxy-methyl-CpG (5hmC; and other oxidized bases) and subsequently removed by a base-excision repair (BER) pathway to re-introduce an unmodified cytosine (Kohli and Zhang, 20I3). DNA demethylation is essential and it plays a predominant role in resetting gene expression in early embryos, in developing germ cells, and in adult tissues (Guibert and Weber, 20l3; Kohli and Zhang, 20I3; Weng et al., 20I3; Messerschmidt et al., 20l4).

Promoter CpG density is central to determining how DNA methylation will affect gene expression. Most high-density CpG promoters (HCP), CpG islands, are unmethylated in all tissues regardless of the level of expression of their associated gene (Lister et al., 2009). Low-density CpG promoters (LCP) are mostly methylated, to different extents, regardless of gene activation or repression. On the contrary, methylation of intermediate CPG-density promoters (ICP) correlates with gene silencing, and these promoters have a greater capacity to acquire lineage-specific DNA methylation patterns. Methylation of HCP promoters, during cell differentiation or tumorigenesis, also directly correlates with gene repression and coincides with the presence of repressive histone marks including histone $\mathrm{H} 3$ lysine 9 methylation and histone deacetylation (Fournier et al., 20I2). It has been postulated that dedicated proteins might bind selectively, and with high affinity to methylated CpG-rich sequences, and participate in the recruitment of chromatin remodeling repressor complexes to silence target genes (Bartke et al., 20 I0; Joulie et al., 20 I0; Fournier et al., 20 I 2; Spruijt et al., 20I3). Conversely, active promoters and enhancers acquired chromatin marks, such as histone $\mathrm{H} 3$ lysine 4 monomethylation (at enhancers) or trimethylation (at promoters) that prevent the activity of DNMTs (Thomson et al., 2010; Clouaire et al., 2012; Zhou et al., 20I2), hence the unmethylated status of most HCP and ICP promoters in the genome (Fig. 2).

The recent advances in the field had a significant impact on our understanding of the role of DNA methylation in mammals, the enzymatic machineries involved in its regulation and its impact on gene expression in normal and pathological contexts. Yet, how local, sequence-specific, DNA methylation is achieved and remodeled still remains elusive. In the current review, we will focus our discussion on the role of transcription factors and signaling pathways in regulating DNA methylation profiles, and their changes in response to developmental and pathological cues.

DNA Methylation and the Regulation of Tissue-Specific Gene Expression During Cell Differentiation

During development, gene activation and repression are tightly regulated to restrict expression to the correct cell type and correct developmental stage. These regulations are controlled by a complex array of transcription factors, histone modifying enzymes, chromatin remodelers, and DNA methylation. With the advent of genome-wide DNA methylation profiling, a variety of examples have been described over the years showing that DNA methylation is dynamically regulated at tissue-specific promoters and cis-regulatory elements during cell differentiation (Hodges et al., 20I I; Lienert et al., 20I I; Stadler et al., 20I I; Hogart et al., 20I2; Feldmann et al., 20I3; Oda et al., 20I3; Ziller et al., 20I3). A detailed analysis of these differentially methylated regions indicates that they are often short regions of DNA and enriched in transcription factor 


\begin{tabular}{|c|c|c|c|c|c|c|}
\hline & $\begin{array}{c}\text { Promoters I } \\
\text { Enhancers }\end{array}$ & $\begin{array}{l}\text { Tumor } \\
\text { suppressor } \\
\text { genes }\end{array}$ & $\begin{array}{l}\text { Germline } \\
\text { genes } \\
\text { promoters }\end{array}$ & $\begin{array}{l}\text { Imprinted } \\
\text { regions }\end{array}$ & $\begin{array}{c}\text { DNA repeats } \\
\text { ERVs }\end{array}$ & $\begin{array}{c}\text { Peri- } \\
\text { centromeres }\end{array}$ \\
\hline $\begin{array}{c}\text { GAIN OF } \\
\text { METHYLATION }\end{array}$ & $\begin{array}{c}\text { DNMTs } \\
\text { H3 lysine } 9 \\
\text { methylation }\end{array}$ & $\begin{array}{c}\text { DNMTs } \\
\text { Zfp354b } \\
\text { ZNF304 } \\
\text { Znf217 } \\
\text { cMyc } \\
\text { PML-RAR }\end{array}$ & $\begin{array}{l}\text { DNMT3B } \\
\text { E2F6 } \\
\text { Max }\end{array}$ & $\begin{array}{c}\text { Kap1 } \\
\text { Setdb1 } \\
\text { DNMTs } \\
\\
\text { ZFP57 } \\
\text { ZNF274? }\end{array}$ & $\begin{array}{c}\text { Kap1 } \\
\text { DNMT3A } \\
\text { DNMT1 } \\
\\
\text { Zfp809 } \\
\text { Zfp819 } \\
\text { PLZF } \\
\text { Rb } \\
\text { others? }\end{array}$ & $\begin{array}{c}\text { DNMT3B } \\
\text { Lsh } \\
\text { ATRX } \\
\text { ZBTB24? }\end{array}$ \\
\hline $\begin{array}{c}\text { LOSSI } \\
\text { MAINTENANCE } \\
\text { OF } \\
\text { METHYLATION } \\
\end{array}$ & $\begin{array}{c}\text { H3 lysine } 4 \\
\text { methylation } \\
\text { REST } \\
\text { CTCF } \\
\text { TET } \\
\text { BER pathways } \\
\text { KDM2A } \\
\text { Transcription } \\
\text { factors } \\
\text { CXXC factors }\end{array}$ & $\begin{array}{c}\text { BER } \\
\text { pathways } \\
\text { TGF-beta } \\
\text { RON } \\
\begin{array}{c}\text { Transcription } \\
\text { factors }\end{array}\end{array}$ & $\begin{array}{l}\text { H3 lysine } 4 \\
\text { methylation }\end{array}$ & & & \\
\hline
\end{tabular}

Figure 2. Summary of the nuclear factors and epigenetic marks involved in the maintenance of DNA methylation status in different regions of the genome. The table recapitulates our current knowledge on transcription factors, chromatin remodellers and histone marks contributing to the establishment of DNA methylation and its erasure. The information is presented according to genomic features, sharing common regulators, such as promoters/enhancers, tumor suppressor genes, germline gene promoters, imprinted regions, DNA repeats, and retroviral elements and peri-centromeric regions.

binding sites compared to the rest of the genome (Hodges et al., 20I I; Stadler et al., 20I I; Hogart et al., 20I2; Oda et al., 2013; Feldmann et al., 2013; Ziller et al., 2013). This recurrent observation obviously pointed to a function of transcription factor binding events in causing these changes in the DNA methylation profile. Yet in most cases the factors essential for these changes remain elusive, and the molecular mechanisms recruiting DNA methylation machineries or enzymatic activity at these promoters (or enhancers) partially understood. While such differentially methylated cis-regulatory regions have being identified during hematopoietic, stem, neuronal, skin, liver or kidney cell differentiation we will restrict our discussion to three different examples that have been deeply characterized: the differentiation of embryonic stem cells into neuronal progenitors, myogenic differentiation, and $\mathrm{T}$ cell activation.

Our first example is the role of transcription factor REST in embryonic stem cells. Single-base profiling of DNA methylation in murine embryonic stem cells (ESC) and neural progenitors (NP; derived from these ESCs) show that many discrete regions of the genome either gain or lose DNA methylation. A number of these regions overlap with cis-regulatory regions or enhancers (i.e., intermediate CPG content DNA regions), characterized by the presence of dedicated histone marks (e.g., histone $\mathrm{H} 3$ lysine 4 mono-methylation), chromatin regulators (e.g., histone acetylase CBP) and transcription factors (Stadler et al., 20I I; Feldmann et al., 20I3). Analysis of the binding profile of zinc finger protein REST (REI-silencing transcription factor, also known as Neural-Restrictive Silencer Factor, NRSF) in ESC showed that REST occupied most of these enhancers, differentially methylated during NP differentiation (Feldmann et al., 20I3). Using a transgenic approach it was elegantly demonstrated that inactivation of REST induces de novo methylation of the DNA sequences normally bound by REST and conversely the expression of REST in the REST-null background is sufficient to restore the unmethylated state of the DNA (Stadler et al., 20I I). Thus, REST binding is necessary and sufficient to dictate the un-methylated status of the DNA at a number of enhancers in ESC cells. How REST prevents DNA methylation remains less clear. REST may prevent the binding of DNMTs or the spreading of DNA methylation from neighboring regions. Alternatively, REST may recruit the TET family enzymes or other chromatin remodelling complexes to actively protect the DNA from de novo methylation and counteract DNMTs activities. Intriguingly REST is among the most expressed zinc finger factor in ESCs, and might contribute to their pluripotent status (Singh et al., 2008; Buckley et al., 2009; Jùrgensen et al., 2009). Thus one can envision that the downregulation of REST expression noticed at the onset of NP-differentiation will coincide with the hyper-methylation of its targets and will promote a neural progenitor expression program. Conversely we can speculate that NP-specific transcription factors, to be identified, may promote loss of DNA methylation at NP-specific enhancers, establishement of active histone marks and further support differentiation.

Analysis of the DNA sequences losing DNA methylation during the differentiation of ESC into NP will help characterize these transcription factors. Importantly, REST does not bind in ESC all sequences gaining DNA methylation during NP differentiation. Additional transcription factors, including CTCF (CCCTC-binding factor), contribute to the shaping of DNA methylation in mouse embryonic stem cells (Stadler et al., 20I I; Feldmann et al., 20I3; Teif et al., 20I4). For instance, a class of CTCF binding sites (outside CpG islands) gain DNA methylation and acquire a condensed chromatin structure, 
concomittant with CTCF expression down-regulation during NP differentiation (Stadler et al., 20I I; Teif et al., 20I4).

Our second example is the transcriptional regulation of Myogenin, a muscle-specific transcription factor that determines commitment and differentiation of myoblasts into myotubes. The methylation of the promoter of Myogenin (an intermediate CpG content promoter, ICP) substantially decreases during the process of somitogenesis (Palacios et al., 2010). This phenomenon is recapitulated in an in vitro model of muscle cell differentiation (Luo et al., 2009; Palacios et al., 20 I0; Oikawa et al., 20I I). Using this latter model for functional studies it has been shown that the Myogenin promoter is initially methylated and bound by two methyl-CpG binding proteins, ZBTB38 (zinc finger and BTB domain containing 38) and MBD2 (methyl-CpG-binding domain protein 2). At the onset of differentiation, the methylation of the promoter is reduced coincident with Myogenin increased expression. Concomitantly, ZBTB38 protein is degraded by a proteasomedependent pathway and MBD2 binding is inhibited by a focal adhesion kinase (FAK)-dependent phosphorylation event (Luo et al., 2009; Oikawa et al., 20 I I). Intriguingly, FAK enhances the transcriptional activity of myocyte enhancer factor 2 (MEF2) and binding of MEF2 to the Myogenin promoter is required for demethylation of the promoter, establishment of active histone marks and gene expression during muscle cell differentiation (Palacios et al., 2010). It is therefore appealing to speculate that induction of FAK activity at the onset of myotube differentiation may regulate a critical switch in sequencespecific transcription factors present at the Myogenin promoter, coincident with demethylation of the promoter, induction of gene expression, and irreversible commitment into the muscle cell lineage. How DNA demethylation is achieved, and its extent, on the other hand has not been properly studied. Whether it is a passive or an active phenomenon also needs to be elucidated.

The third example is the activation of interleukin (IL)-2 gene expression upon activation of naïve CD4 T cells (by anti-CD3 $\varepsilon$ and anti-CD28). In turn IL-2 drives their proliferation. The activation of IL-2 expression coincides with the demethylation of specific CpGs in the promoter of the gene (Bruniquel and Schwartz, 2003). The study of the kinetic of DNA demethylation showed that it occurs in absence of DNA replication indicating an active, enzymatic, mechanism (Bruniquel and Schwartz, 2003). The demethylation of the promoter is not sufficient to activate IL-2 expression and specific transcription factors including NF-AT (nuclear factor of activated T cells) and OCT-I (octamer-binding transcription factor I) need to bind to the unmethylated promoter in order to transcribe the gene (Murayama et al., 2006). For instance, in resting cells (after antigen withdrawal) the promoter is in an un-methylated state, bound by OCT-I, but IL-2 is not expressed. On the contrary, after re-stimulation with antigen, IL-2 expression is induced and the transcriptional activation of IL- 2 is much faster than in naïve cells, suggesting that the absence of methylation on the IL-2 promoter and OCT-I binding may prime gene expression (Murayama et al., 2006). These data imply that IL-2 expression required demethylation of the promoter upon antigen activation but also binding of a dedicated set of transcription factors. In vitro assays and preliminary data in vivo suggest that active DNA demethylation may be controlled by DNA glycosylase Myh (MUTYH in human) that is strongly transcriptionally induced upon activation of naïve T cells (Wu and Zheng, 20l4). Using a very similar method, it was also reported that IL-4 expression is strongly enhanced upon CD4 T cells activation into Th2 cells. Activation of IL-4 is dependent on GATA-3 accumulation, passive demethylation of the locus and release of the methyl-CpG binding protein MBD2 and DNA methyltransferase DNMTI from the locus (Hutchins et al., 2002; Makar et al., 2003).
Cell fate decisions require (at least) temporal co-expression of tissue-specific genes and environmental stimulation. This regulation primarily orchestrates a switch in the transcriptional program and it is likely that transcription factors, by recruiting DNMTs or demethylating activities, may ensure that tissuespecific gene expression and cell identity are stably maintained during later cell divisions. Consistent with such an interpretation, tissue-specific genes are associated with intermediate CpG-content promoters (ICP), whose level of methylation is strongly correlated with their level of expression (Lister et al., 2009; Joulie et al., 20 I0; Fournier et al., 2012; Ziller et al., 20I3). The variety of tissu-specific genes, hence promoter sequences, on the other hand, suggests that many transcription factors might be required to properly establish tissu-specific DNA methylation profiles. Accordingly, using a protein array analysis Pierre-François Cartron and colleagues have identified a large variety of transcription factors that can interact with DNMTs, and thus probably alter DNA methylation at target genes upon cell differentiation (Pacaud et al., 2014) (Fig. 2).

DNA Methylation and the Silencing of Germline Genes in Somatic Tissues

Germline genes are normally only expressed in germ cells. The regulation of their expression represents a unique example of regulation that primarily relies on DNA methylation. Studies have shown that, in germ cells, CpG-island promoters at germline genes are unmethylated and associated with a chromatin structure characteristic of active gene expression. On the contrary, in somatic tissues, promoters at germline genes are fully methylated and the genes transcriptionally silenced (De Smet and Loriot, 2013). Intriguingly, global hypomethylation in cancer lead to the recurrent activation of a limited number of genes, most of them germline genes. Germline genes are therefore often used as tumor-specific antigens for diagnostic applications and for translational applications. Yet, very little is known on the mechanisms involved in the deposition and maintenance of DNA methylation at these peculiar CpG-island promoters.

An elegant study, by Claire Francastel's laboratory, showed that DNA methylation at several germline genes is altered in a mouse hypomorphic Dnmt3b background and in a null background for transcription factor E2F6. Complementary experiments demonstrated that the protein $\mathrm{E} 2 \mathrm{~F} 6$ is required for Dnmt3b binding at these germline gene CpG-island promoters in somatic tissues (Velasco et al., 2010). Intriguingly, follow up studies showed that E2F6 interacts with DNMT3B (Pacaud et al., 20I4) and that E2F6-binding elements, predicted in silico, are detected at germline gene promoters (Borgel et al., 2010). Yet it remains to be formally shown that this interaction leads to the establishment or to the maintenance of DNA methylation. In addition, many E2F6 targets have been described in mouse and human cell lines and most of them are not regulated by DNA methylation (Project Consortium., 20I2). It is therefore likely that additional transcription factors help target Dnmt3b at these promoters or that a dedicated E2F6 complex, to be characterized, bind at these promoters together with Dnmt3b (Fig. 2). A good candidate is the cMyc partner, Max (Maeda et al., 2013). Its inactivation in mouse embryonic stem cells causes the reactivation of some germline genes, among other genes (Maeda et al., 2013).

\section{DNA Methylation and the Regulation of Mono-Allelic Expression}

DNA methylation plays an essential role in genomic imprinting or mono-allelic expression, the process that causes genes to be expressed only from one parental allele (Hanna and Kelsey, 
20I4). Over a hundred imprinted genes have been identified and many of these genes are organized in clusters controlled by the differential allelic DNA methylation of a CpG-rich sequence called the imprinting control region (ICR). These regions acquire a parental origin-specific differential methylation in the male or female germline, escape a genomewide wave of methylation reprogramming during the first days of embryonic development and retain parent-of-origin DNA methylation in the adult. Mono-allelic expression is essential for normal growth and development. Disruption of imprinting may lead to serious developmental disorders (e.g., Prader-Willi syndrome, Angelman syndrome, Beckwith-Wiedemman syndrome) and is described in cancer (e.g., Wilm's tumor) (Lim and Maher, 2010; Hanna and Kelsey, 2014).

Among the factors involved in imprinting, the zinc finger transcription factor Zfp57 is responsible for the maintenance of DNA methylation imprints (Fig. 2). Inactivation of the maternal and zygotic Zfp57 in the mouse results in the loss of differential DNA methylation and genomic imprints ( $\mathrm{Li}$ et al., 2008). Zfp57 encodes a Kruppel-associated box-containing zinc-finger protein (KRAB-ZNF) factor. Previous studies indicated that the KRAB domain interacts with corepressor protein KAPI (also known as TRIM28 and TIFIbeta), a docking protein for heterochomatin protein I, histone $\mathrm{H} 3$ lysine 9 methylases, and DNA methyltransferases (Quenneville et al., 20I2). A molecular analysis of Zfp57 function in embryonic stem cells indicated that the protein binds to the methylated allele of ICRs and recruits KAPI and associated co-factors onto the methylated allele. Inactivation of Zfp57 in ESC alters DNA methylation, chromatin marks and allelic expression at a number of imprinted regions (Quenneville et al., 20I I). Intriguingly, human ZFP57 functionally replaces mouse Zfp57 in embryonic stem cells and mutation in human ZFP57 affect DNA methylation at a subset of imprinted loci in individuals suffering transient neonatal diabetes (Mackay et al., 2008; Takikawa et al., 20I3). Altogether these studies suggested a conserved role for Zfp57 in the maintenance of DNA methylation pattern at imprinted loci in mammals, despite clear differences in early embryonic developmental processes.

A genome-wide analysis of Zfp57 targets in ESC indicated that Zfp57 is not selectively binding to ICR regions in the genome (Quenneville et al., 20I I). Nevertheless, it binds together with KAPI and the histone $\mathrm{H} 3$ lysine 9 methylase SETDBI only to these ICR regions, suggesting that a dedicated Zfp57/KAPI/SETDBI complex ensures imprinting in mouse ESC cells. Analysis of these DNA sequences highlighted the presence of a common methylated hexameric TGC ${ }^{\mathrm{m}} \mathrm{CGC}$ sequence motif (Quenneville et al., 20I I). In vitro Zfp57 binds to this methylated motif with stronger affinity than the unmethylated counterpart DNA. This latter result strongly supported the role of $\mathrm{Zfp} 57$ in targeting the methylated parental allele in vivo to maintain parent-of-origin methylation and expression. How Zfp57 DNA selectivity is achieved is still unclear. Studies will be necessary to understand why the corepressors KAPI and SETDBI only stably interact with Zfp57 at these imprinted regions.

Targeting of the KRAB domain alone is sufficient to induce transcriptional silencing and DNA methylation at the targeted locus, even after removal of the transcriptional repressor, in embryonic stem cells (Quenneville et al., 20I2). This effect however is not observed in adult differentiated cells where $\mathrm{KRAB}-$ mediated gene silencing is not associated with DNA methylation (Quenneville et al., 20I2). Thus, thanks to its ability to bind an ICR-specific methylated-sequence and to attract the KAPI/SETDBI/DNMTs complex, Zfp57 is key in the establishment and/or maintenance of DNA methylation pattern at many imprinted loci, in a timely manner, during early mouse embryonic development. Importantly, during development, the other parental allele should remain unmethylated to perpetuate the differential methylation at ICRs. It is noteworthy to mention that this phenomenon might actually be the result of an active protection mechanism involving transcription factors, chromatin remodelling activities and TET activities.

Zfp57 is only expressed in embryonic stem cells suggesting that other KRAB-ZNF factors (over 400 in the human genome) might be involved in the maintenance of DNA methylation during later developmental stages and in tissues in which Zfp57 is not expressed. Consequently, another KRAB-ZNF factor, ZNF274, emerged as a regulator of parent-of-origin expression. SNORDI 16, a non-coding RNA contained in the bi-partite Prader-Willi syndrome imprinting center on chromosome 15 in human, is mono-allelically expressed (Cruvinel et al., 20I4). Chromatin immunoprecipitation analysis showed that ZNF274 binds specifically to the methylated maternal allele of the ICR in the SNORD I 6 locus. ZNF274 interacts with KAPI and SETDBI, and these latter factors are recruited to the ZNF274-bound DNA at the SNORDI 16 locus (Cruvinel et al., 20I4). Yet, ZNF274 also binds to other genomic sites in the human genome that are not subjected to imprinting, and these sites also show significant levels of KAPI and SETDBI (Frietze et al., 2010). It thus remains to be properly shown that ZNF274 dictates DNA methylation at the SNORD I I 6 ICR and elucidate how ZNF274 recognizes the ICR. In conclusion, the data still remain sparse to clearly state on the role of ZNF274 in the control of imprinting but, owing to the presence of the KRAB domain and the resemblance with the molecular function of ZFP57, one can suggest a probable similar function.

Other transcription factors have been described to bind to ICRs. Their functions in the control of imprinting and in the establishment or maintenance of the methylation pattern at these ICRs await further investigation. For instance, chromatin immunoprecipitation analysis in chimeric mice indicates that ZBTB38 and ZBTB4 bind to the methylated allele of the InsulinLike Growth Factor 2 (IGF2) ICR, a growth-promoting hormone during gestation (Filion et al., 2006). Intriguingly, different single-nucleotide polymorphisms in the ZBTB38 locus, correlated with changes in ZBTB38 expression, have been linked to human height phenotype (Gudbjartsson et al., 2008). The ZBTB38 gene, like IGF2, may thus be involved in determining growth, and ZBTB38 might do so by regulating IGF2 mono-allelic expression.

\section{DNA Methylation and the Silencing of Repetitive} Elements and Endogenous Retroviral Elements

It is well recognized that DNA methylation represents the primary mechanism of transposition repression in the host genome. In the absence of DNA methylation, DNA repeats and endogenous retroviral elements are re-activated and they invade the host genome leading to a toxic cellular response (Goff, 2009; Rowe et al., 2010; Tan et al., 2013; Wolf and Rowe et al., 20I3). Yet, how this methylation is deposited on newly integrated transposable elements and how it is maintained in subsequent cell divisions remains a matter of debate.

A very elegant series of experiments from Didier Trono's laboratory indicated that this regulation might primarily rely on the co-repressor KAPI. The study of gene expression regulation in mouse (tissues and embryonic stem cells) and in human samples inactivated for KAPI showed a strong reactivation of multiple DNA repeats, notably endogenous retroviral elements (Rowe et al., 2010, 20I3; Lukic et al., 20I4; Turelli et al., 20I4). In addition, analysis of KAPI binding in ESC cells showed an enrichment at a number of endogenous retroviral elements and mutation of KAPI-residues involved in KRABdomain binding abolished this binding preference (Lukic et al., 20I4). The concomittant identification of the role of KRAB-ZNF 
factors in de novo methylation and the rapid expansion of this family of ZNFs in mammalian genomes, led to the hypothesis that KRAB-ZNF factors might rapidly evolve to provide a repertoire of $\mathrm{KAPI}$-interacting DNA binding proteins and counteract accumulation in the genome of foreign transposable elements (Lukic et al., 20I4). Consistent with this provocative hypothesis inactivation of at least two different KRAB-ZNF factors causes the reactivation of different classes of DNA repeats in human and mouse cells (Li et al., 2008; Wolf and Goff, 2009; Tan et al., 20I3).

Embryonic stem cells have been shown to block retrovirus replication. For example, infection of stem cells by the murine leukemia virus results in its integration in the genome but the retrovirus is then transcriptionally silenced. This phenomenon required specific cis-regulatory element in the viral genome and the recruitment of the KAPI corepressor (Wolf and Goff, 2007, 2009). A proteomic screen, identified two KRAB-ZNF proteins, ZFPI 24 and ZFP809, bound onto the viral DNA but only ZFP809 could specifically bound, on its own, to the sequence in vitro (Wolf and Goff, 2009). Functional analyses in ESC cells next showed the key function of ZFP809 in recruiting the KAPI/DNMTs complex to silence the murine leukemia virus (Wolf and Goff, 2009). In another study, inactivation of Zfp8I9 in mouse embryonic stem cells causes the reactivation of certain endogenous retroviral elements (Tan et al., 20l3).

Integration of gene expression profiling and histone marks and transcription factors mapping provided strong evidence that repeated DNA sequences are important regulatory elements in genomes (ENCODE) Project Consortium., 2012. As such, they can serve as alternative promoter of genes, enhancers or insulators, among other things. It is thus likely that silencing of these DNA repeats is achieved by, redundant, alternative chromatin remodelling complexes and pathways. Consequently, many functional binding sites for transcription factors, including NFY (nuclear transcription factor Y), STATI (signal transducer and activator of transcription I) and CTCF, are experimentaly identified in these repeated DNAs [Project Consortium., 2012]. Yet, in most cases the studies focus on the role of these transcription factors in altering the epigenetic signature or expression level of neighboring host genes. It thus remains to be investigated whether these transcription factors also play a role in the control of repeated DNA silencing and DNA methylation (Fig. 2).

For instance, PLZF (promyelocytic leukaemia zinc finger protein, also known as ZBTB I6) plays a key role in the maintenance of germ cells in adult tissue and hematopoietic stem cells (Puszyk et al., 20I3). An analysis of DNA methylation profile in bone narrow and testis of mice bearing a DNAbinding inactivating mutation in PLZF identify a hundred of regions differentially methylated, most of them overlapping with a LINEI (LI) retrotransposon (Puszyk et al., 20I3). ZBTBI 6 targets these $L I$ elements by interacting both with the DNA and the LI RNA, leading to the recruitment of DNMTI and silencing of LI retro-transposition (Puszyk et al., 2013). Intriguingly, retinoblastoma protein $(\mathrm{Rb})$ also represses $\mathrm{LI}$ elements and it was previously shown that it interacts with ZBTBI 6 (Petrie et al., 2008). ZBTBI 6 regulates the expression of both protein-coding genes and DNA repeats. The implication of Rb in both functions highlighted the implication of a common regulatory complex. It also emphasis that the implication of transcription factors in DNA repeats silencing might have been previously overlooked.

Inhibition of DNMT3A phosphorylation also causes the reactivation of many DNA repeats, independently of DNMTI and DNMT3B. DNMT3A is a bona-fide substrate for the protein kinase CK2 (Deplus et al., 20I4). CK2 phosphorylate DNMT3A at serines 386 and 389 in vivo and in vitro (number according to the mouse protein). Surprinsingly this phosphorylation reduces the de novo methylase activity of DNMT3A in vitro. Yet, in vivo the effects on DNA methylation are context specific, as shown in human cancer cells inactivated for CK2. In unique portions of the human genome no significant change in DNA methylation are detected, while at a subset of DNA repeats both significant hypermethylation (e.g., Alu elements) and hypomethylation (e.g., LINE, LTR, and Satellite repeats) is observed. At the molecular level the phosphorylation of DNMT3A by CK2 controls its subnuclear distribution. Phosphorylated DNMT3A is mostly detected in heterochromatin domains, while dampening the phosphorylation relocates DNMT3A in the euchromatic compartment of the nucleus. Whether the phosphorylation affects the binding of DNMT3A to specific heterochromatic proteins or transcription factors binding satellite DNAs is an exciting hypothesis that will require further investigation.

DNA Methylation and the Silencing of Tumor Suppressor Genes in Cancer

In cancer, a global hypo-methylation of the genome coincides with the development of the disease (Zane et al., 20I4). Conversely several CpG islands, including those linked to cancer specific genes affecting cellular growth control, gain methylation and they are stably silenced (Gazin et al., 2007; Thillainadesan et al., 2012; Wajapeyee et al., 2013; Serra et al., 20I4). It was though that the process may be stochastic and that errors in the fidelity of the DNA methylation machinery would randomly cause hyper-methylation of a CPG island of a cancer gene. An alternative model, that regain interest, postulate that oncogenic signaling drives this epigenetic repression in cancer by regulating the function of transcription factors able to attract DNMTs to a CpG island of a cancer gene (Fig. 2).

Using an in vitro system recapitulating the hyper-methylation and silencing of the Fas gene by oncogenic Ras (Kras), Michael Green's laboratory performed a genetic screen to identify the cellular components required for the silencing of Fas (Gazin et al., 2007). They identified 28 factors involved in the process including: the maintenance methyltransferase DNMTI, a serinethreonine kinase PDPKI, different chromatin remodelers (RCOR2, ASFIA, TRIM66/TIFID, DOTIL, EZH2, SMYDI and others) and several sequence-specific transcription factors (ZFP354B, ZCCHC4, and SOXI4). Using chromatin immunoprecipitation assays they confirmed the direct binding of these transcription factors and chromatin remodelers at the Fas promoter in response to expression of activated Ras. Individual inactivation of several of these 28 genes impairs the binding of DNMTI at the Fas promoter and the hyper-methylation of the promoter in response to expression of activated Ras (Gazin et al., 2007). In a follow-up study, the same group studied the hierarchy of these 28 factors. They provided strong evidence that the binding of KRAB-ZNF ZFP354B at the Fas promoter is the key event leading to further assemble of transcriptional corepressors and chromatin modifying enzymes and eventually DNMTI to the promoter (Wajapeyee et al., 20I3). An analysis of additional cancer genes (Sfrpl, Par4, Plagll, H2-KI, and Lox) indicates that several of the 28 genes are also required for the silencing of these five genes upon expression of activated Ras (Gazin et al., 2007). At least in the case of Sfprl, ZFP354B binding also preceded co-repressors, chromatin-modifying enzymes and DNMTI binding (Wajapeyee et al., 20I3). Thus activated Kras may direct epigenetic silencing at a number of cancer gene relying on the binding of ZFP354B at their promoters.

Using a similar approach, the same laboratory also investigated the hyper-methylation and silencing of the tumor suppressor genes pI $4^{\mathrm{ARF}}$ in colorectal cancer (Serra et al., 20।4). They identified eight genes essential for the transcriptional repression of $\mathrm{p} / 4^{\mathrm{ARF}}$ in DLDI human colorectal 
cancer cells that contain an activated KRAS mutation. Among the proteins they identified is the KRAB-ZNF ZNF304 factor. Using a variety of assays, they demonstrated that ZNF304 binds to the PI $4^{\mathrm{ARF}}$ promoter in different colorectal cancer cell lines and also KRAS-positive human colorectal tumor samples. Inactivation of ZNF304 causes the re-activation of p I4 $4^{\mathrm{ARF}}$ expression and the demethylation of the promoter. Surprisingly, other tumor suppressor genes, including $\mathrm{P} / 6^{\mathrm{INK} 4 \mathrm{~B}}$ (CDKN2B) and pI $5^{\mathrm{INK} 4 \mathrm{~A}}$ (CDKN2A) are also epigenetically silenced by ZNF304 in colorectal cancer cells containing an activated Ras mutation.

Together, these studies indicate that activation of Ras perturbes intracellular signalization leading to the accumulation of KRAB-ZNF factors ZFP354B and ZNF304 in a cancer specific fashion. In turn, these KRAB-ZNF factors specifically bind to unmethylated $C_{p}$ G-rich islands to initiate the recruitment of co-repressors, chromatin remodelers and DNMTI. ZFP354B accumulates in cancer cells because of its transcriptional upregulation and its phosphorylation by Rasactivated kinase PDKPI (Gazin et al., 2007). ZNF304 accumulation in CRC is caused by Ras-induced inactivation of deubiquitinase USP28 (Serra et al., 20I4).

Tumor suppressor gene pI $5^{\text {INK } 4 A}$ is hypermethylated and silenced in breast cancer. Using MCF7 breast cancer cells, it was demonstrated that hypermethylation of the pI $5^{\text {INK4A }}$ promoter is dependent on the zinc finger protein ZNF2I 7 (Thillainadesan et al., 20I2). ZNF2I7 overexpression has been observed in many cancer types, including breast cancer, and it is often linked to immortalization, increased proliferation, and resistance the TGF-beta, a cytokine that trigger a complex antiproliferative cellular response. In MCF7 cells, ZNF2I 7 is responsible for the targeting of the co-repressor complex coREST and DNMT3A to the pI $5^{\text {INK4A }}$ promoter, its hypermethylation and its silencing (Thillainadesan et al., 20I2). Surprisingly, the antiproliferative action of TGF-beta involved the demethylation of the pI $5^{\mathrm{INK} 4 \mathrm{~A}}$ promoter and the reactivation of the gene. Study of this mechanisms in MCF7 cells indicate that TGF-beta causes the disassembly of the ZNF2 I / /coREST/DNMT3A complex from the pI INK4A promoter in a few minutes and the concomitant recruitment of transcription factor SMADs, of co-activators CBP and of BER pathway factors AID (activation-induced deaminase) and TDG (thymidine DNA glycosylase) (Thillainadesan et al., 20I2).

Epigenetic silencing in cancer also involves non-ZNF transcription factors. Methylation and silencing of tumor suppressor gene RASSFI is dependent on CMYC and Polycomb-repressor proteins (Palakurthy et al., 2009). Intriguingly, DNMT3B physically interacts with PcG-proteins and with cMYC in the nucleus (Viré et al., 2006). In acute promyelocytic leukemia the oncogenic fusion transcription factor PML-RAR binds the retinoic acid receptor beta-2 promoter and induces its transcriptional silencing, through recruitment of DNMT3A and de novo methylation of the promoter (Di Croce et al., 2002). Again, multiple transcription factors, some of them mys-regulated or mutated in cancer, interact with DNMTs in vitro, and may contribute to aberrant changes in DNA methylation in cancer (Pacaud et al., 2014) (Fig. 2). A key question remaining is to understand how the deregulation (or mutation) of these transcription factors can cause very selective changes in the DNA methylation profile that will promote tumorigenesis. Is this information embedded in the promoter sequence of tumour suppressor genes?

\section{Perspectives in the Field of DNA Methylation}

Many transcription factors have been shown to control DNA methylation status of their target sites. Among them, many belongs to the KRAB-ZNF family (Gazin et al., 2007; Li et al., 2008; Wolf and Goff, 2009; Oikawa et al., 20I I; Feldmann et al.,
2013; Puszyk et al., 2013; Wajapeyee et al., 2013; Cruvinel et al., 20I4; Serra et al., 20I4). This later observation may relate to the observation that the isolated $\mathrm{KRAB}$ domain by associating with KAPI/DNMTs lead to de novo methylation at target sites in stem cells (Quenneville et al., 20 I2). It was therefore postulated, and later strongly experimentally supported, that KAPI/DNMTs control the maintenance of DNA methylation, independently of DNA replication, on a number of genomic targets. Yet, DNMTs have been shown to be recruited onto the chromatin by other chromatin remodelers, such as SETDBI or G9a, or secondary to gene silencing (Gibbons et al., 2000; Dennis et al., 200I; Guibert and Weber, 20I3; Pacaud et al., 20I4). Thus, only the identification of the full-spectrum of transcription factors involved in the regulation of DNA methylation will tell whether this function is predominantly confer to KRAB-ZNF factors. This systematic analysis might help understand why only a limited number of factors per family are involved in the shaping of DNA methylation. In the case of ZNF factors several explanations have been postulated. The resolution of the structure of the ZNF fingers of Zfp57 bound onto methylated DNA indicated that a specific amino-acid sequence in the DNA binding ZNF fingers might be required for the recognition and binding of methylated CpG sequences (Liu et al., 20I2; BuckKoehntop and Defossez, 2013). Using this knowledge, researchers have postulated that ZNF factors containing this motif might likely contribute to shape DNA methylation profile (Liu et al., 20I3). An alternative hypothesis rely on the observation that KRAB-ZNF factors are present uniquely in vertebrate genomes and have expanded quite dramatically in mammalian genomes. As DNA repeats sequences also quickly evolved in mammalian genomes, it is suggested that humanspecific KRAB-ZNF factors might primarily contribute in DNA repeats silencing (Lukic et al., 20I4).

Another open question, despite intense scrutiny, is the nature of the factors involved in the establishment of DNA methylation at centromeres and telomeres. We still do not know how the DNMTs are targeted to these regions of the genome. Demethylation of pericentromeric satellite type II and III repeats, caused by loss-of-function mutations in DNMT3B, is an important feature of the chromatin in Immunodeficiency, Centromere instability and Facial anomalies (ICF) syndrome patients (Nitta et al., 20I3; Weng et al., 20I3). Lessons from genetic studies of the causes of ICF indicate that around 50$60 \%$ of cases are explained by inactivating mutations in DNMT3B coding sequence. Yet, in the remaining patients no mutation was identified in the coding sequence of DNMT3B. The advent of exome sequencing technologies lead to the identification of mutations in a ZNF factor ZBTB24 in a category of ICF patients (Nitta et al., 20I3). In mouse cells, ectopically expressed ZBTB24 and DNMT3B are targeted to pericentromeric heterochromatin compartments,

independent of the DNA methylation status (Nitta et al., 20I3). Some of the mutations, identified in ICF patients, lies in a wellconserved zinc finger of ZBTB24 and impair the recruitment of ZBTB24 onto pericentromeric DNA in mice cells (Nitta et al., $2013)$. It is therefore very tempting to speculate that ZBTB24 is responsible for the recruitment of DNMT3B at pericentromeric DNAs and the establishment and/or maintenance of their methylation status. Two helicases, ATRX (alpha thalassemia/mental retardation syndrome $X$-linked) and Lsh (Lymphoid-specific helicase; HELLS in human), are critically required for the maintenance of DNA methylation at pericentromeric regions in mouse cells (Gibbons et al., 2000; Dennis et al., 200I). These helicases lack a dedicated DNA binding domain and their recruitment at pericentromeric DNA has remained elusive for years. It would be tempting to speculate that ZBTB24 might contribute to target these helicases. Further studies will be required to clarify ZBTB24 function at centromeres. 
Local changes in DNA methylation have been noticed in other context than cancer. For instance, neuronal activity induces local and specific changes in DNA methylation in the brain (Martinowich et al., 2003; Murgatroyd et al., 2009). Aberrant DNA methylation has been described in patients subject to a number of psychotic and neurodevelopmental disorders, including alzheimer disease, schizophrenia, anxiety, and cognitive deficits (Weng et al., 2013). Conversely, drugs used to manage some of these diseases reshape DNA methylation in neurons (Dong et al., 2008). Surprinsingly, some of these changes might occur very early in the development of the individual, far before the clinical signs of the disease are manifest in the adult (Martinowich et al., 2003; Borrelli et al., 2008; Murgatroyd et al., 2009; Guo et al., 20I I; Weng et al., 2013). In mice, maternal care, such as licking and grooming, influences the expression of glucocorticoid receptor (GR) in the neurons of the hypothalamus in the pups, and later in the adult (Weaver et al., 2007). GR is a major component of the stress response in the brain, and is implicated in short and long-term adaptations in response to stress (Dong et al., 2008; Murgatroyd et al., 2009). Maternal care causes the up-regulation of GR expression in the brain of pups, its maintenance in the adult and a lower propensy to develop depression-like phenotypes compared to pups deprived of maternal care. At the molecular level, maternal care induces the expression of transcription factor NGFI-A in the hypocampal neurons. In turn, NGFI-A binds to the GR promoter, induces its demethylation and its increased expression, that will be maintained later in life (Weaver et al., 2007).

Lessons gathered from the study of de novo DNA methylation events might likely apply to the DNA demethylation field. Transcription factors may play an important role in triggering local DNA demethylation events. For instance, in cancer, overexpression of the receptor tyrosine kinase RON (also known as macrophage stimulating I receptor, MSTIR) in a number of human cancers correlates with metastasis and poor prognosis (Cunha et al., 2014). In a cellular model it was shown that overexpression of RON lead to hypermethylation and hypomethylation of a number of intergenic regions, and causes a better propensy to form metastasis when injected in the mice (Cunha et al., 2014). In a complementary analysis it was shown that RON triggers the accumulation of the DNA glycosylase MBD4 and that this regulation drives metastasis and part of the changes in DNA methylation. Yet, MBD4 lacks a DNA binding domain, and its selectivity might be mediated by dedicated transcription factors or histone marks (Hendrich et al., 1999; Laget et al., 2014).

\section{Concluding Remarks and Emerging Concepts}

DNA methylation plays an important role in the control of gene expression and cell fate in mammals. Its regulation and function has been upon intense scrutiny since its discovery in mid-1900s. Yet, how DNA methylation patterns are established during embryogenesis, and edited in adult tissue, remains a matter of intense debate. Profiling of DNA methylation in many cell type, species and environmental set up indicates that the DNA methylation profile is thighly correlated with the cell type and its environment. As a consequence, de novo methylation and DNA demethylation events are not randomly distributed but are actually targeted to particular regulatory DNA elements in the genome, including promoters, enhancers or repeated DNAs. For this latter reason researchers have focused on the role of transcription factor in these DNA methylation events.

Yet, it is also recognized that non-coding RNAs, short and long, contribute to the establishment and editing of DNA methylation profiles in mammals. Non-coding RNAs may directly interact and control methylation and demethylation activities and, as a consequence, the pattern of DNA methylation in the genome (Di Ruscio et al., 2013; Arab et al., 20I4; Castro-Diaz et al., 20I4; Molaro et al., 20I4; Turelli et al., 20l4). For instance, antisense long non-coding RNA TARID (TCF2I antisense RNA inducing demethylation), activates TCF2I expression by inducing promoter demethylation. TARID sequence is complementary to the sequence of the TCF2 I promoter. Its transcription causes the anchoring of GADD45A (growth arrest and DNA-damageinducible, alpha), a regulator of DNA demethylation, at the TCF2I promoter and its subsequent chromatin remodelling (Arab et al., 2014). Understanding the interplay between noncoding RNAs and transcription factors in the establishment and the maintenance of DNA methylation is therefore an important challenge for the future.

The characterization of the factors underlying the dynamic nature of DNA methylation and its plasticity with regards to environmental cues highlight potential pathways leading to changes in DNA methylation and how this information might be used for translational applications. For instance, in cell-based assays, it is possible to reprogram the methylation level of a CPG island by targeting a TET-fusion to the given CPG island (Zhang et al., 20I2; Maeder et al., 20I3). With the development of sophisticated systems of genome engineering, one can imagine in the future to use such strategies to revert aberrant methylation at a specific locus and treat a disease (with all the ethical issues such approaches will raised). Alternatively, inactivation of the transcription factor (or signaling pathways) responsible to the maintenance of aberrant DNA methylation pattern could prove useful to selectively target cancer cells. In biotechnology and industry also the gains could be immense. For instance, cell reprogramming is facilitated by addition of vitamin $\mathrm{C}$, a compound that alters the activity of TET enzymes and DNA methylation maintenance (Blaschke et al., 2013; Chen et al., 20I3). In the future one can imagine better control cell reprogramming by adding to the cocktail of reprogramming transcription factors (the 4 "Yamanaka" factors: KLF4, OCT3/4, SOX2, c-MYC), compounds that will modulate the activity of transcription factors involved in DNA methylation maintenance.

The identification of transcription factors as important drivers of changes in DNA methylation pattern in disease and during development offer tremendous opportunities in the future in basic research, in human medicine and in therapeutic applications.

\section{Acknowledgements}

BM and CM wish to thank Pierre-Antoine Defossez, Guillaume Velasco, Claire Francastel and the members of the Epigenetic and Cell Fate laboratory for support, discussions and suggestions during the writing of the manuscript. BM and CM also like to apologize that due to space limitations only a limited number of research papers could be cited. BM work is supported by the CNRS, a grant from Ligue contre le Cancer (Comité de Paris) and a Marie Curie International Reintegration Grant (PIRG07-GA-20 I0-268448). CM is a recipient of $\mathrm{PhD}$ fellowship from the Ministère de l'enseignement supérieur et de la recherche (MESR).

\section{Literature Cited}

Arab K, et al. 2014. Long noncoding RNA TARID directs demethylation and activation of the tumor suppressor TCF2I via GADD45A. Mol Cell 55:604-6I4.

Bartke T, et al. 2010. Nucleosome-interacting proteins regulated by DNA and histone methylation. Cell 143:470-484.

Blaschke K, et al. 2013. Vitamin C induces Tet-dependent DNA demethylation and a blastocyst-like state in ES cells. Nature 500:222-226.

Borgel J, et al. 2010. Targets and dynamics of promoter DNA methylation during early mouse development. Nat Genet 42:1093-1100.

Borrelli E, Nestler EJ, Allis CD, Sassone-Corsi P. 2008. Decoding the epigenetic language of neuronal plasticity. Neuron 60:961-974.

Bruniquel D, Schwartz RH. 2003. Selective, stable demethylation of the interleukin-2 gene enhances transcription by an active process. Nat Immunol 4:235-240. 
Buck-Koehntop BA, Defossez P-A. 2013. On how mammalian transcription factors recognize methylated DNA. Epigenetics 8:13I-137.

Buckley NJ, Johnson R, Sun Y-M, Stanton Is LW. 2009. REST a regulator of pluripotency? Nature 457:E5-E6.

Castro-Diaz N, et al. 2014. Evolutionally dynamic LI regulation in embryonic stem cells. Genes Dev 28:1397-1409.

Chen J, et al. 2013. H3K9 methylation is a barrier during somatic cell reprogramming into iPSCs. Nat Genet 45:34-42.

Clouaire T, et al. 2012. Cfpl integrates both CPG content and gene activity for accurate H3K4me3 deposition in embryonic stem cells. Genes Dev 26:1714-1728.

Cruvinel E, et al. 2014. Reactivation of maternal SNORD I 16 cluster via SETDBI knockdown in Prader-Willi syndrome iPSCs. Hum Mol Genet 23:4674-4685.

Cunha S, et al. 2014. The RON receptor tyrosine kinase promotes metastasis by triggering MBD4-dependent DNA methylation reprogramming. Cell Rep 6:14I-I54.

De Smet C, Loriot A. 20 I3. DNA hypomethylation and activation of germline-specific genes in cancer. Adv Exp Med Biol 754:149-166.

De la Rica L, et al. 2013. PU. I target genes undergo Tet2-coupled demethylation and DNMT3b-mediated methylation in monocyte-to-osteoclast differentiation. Genome Bio I4:-R99.

Dennis K, Fan T, Geiman T, Yan Q, Muegge K. 200I. Lsh, a member of the SNF2 family, is required for genome-wide methylation. Genes Dev 15:2940-2944.

Deplus R, et al. 2014. Regulation of DNA Methylation Patterns by CK2-Mediated Phosphorylation of Dnmt3a. Cell Rep 8:743-753.

$\mathrm{Di}$ Croce L, et al. 2002. Methyltransferase recruitment and DNA hypermethylation of target promoters by an oncogenic transcription factor. Science 295:1079-1082.

Di Ruscio A, et al. 2013. DNMTI-interacting RNAs block gene-specific DNA methylation. Nature 503:37I-376.

Dong E, Nelson M, Grayson DR, Costa E, Guidotti A. 2008. Clozapine and sulpiride but not haloperidol or olanzapine activate brain DNA demethylation. Proc Natl Acad Sci U.S.A 105:13614-13619.

Feldmann A, et al. 2013. Transcription factor occupancy can mediate active turnover of DNA methylation at regulatory regions. PLoS Genet 9:-el003994.

Filion GJP, et al. 2006. A family of human zinc finger proteins that bind methylated DNA and repress transcription. Mol Cell Biol 26:169-181.

Fournier A, Sasai N, Nakao M, Defossez P-A. 2012. The role of methyl-binding proteins in chromatin organization and epigenome maintenance. Brief Funct Genomics 1 1:25I-264.

Frietze S, O'Geen H, Blahnik KR, Jin VX, Farnham PJ. 2010. ZNF274 recruits the histone methyltransferase SETDBI to the 3' ends of ZNF genes.. PLoS ONE 5:el5082.

Gazin C, Wajapeyee N, Gobeil S, Virbasius C-M, Green MR. 2007. An elaborate pathway required for Ras-mediated epigenetic silencing. Nature 449:1073-1077.

Gibbons RJ, et al. 2000. Mutations in ATRX, encoding a SWI/SNF-like protein, cause diverse changes in the pattern of DNA methylation. Nat Genet 24:368-371.

Gudbjartsson DF, et al. 2008. Many sequence variants affecting diversity of adult human height. Nat Genet 40:609-615.

Guibert S, Weber M. 2013. Functions of DNA methylation and hydroxymethylation in mammalian development. Curr Top Dev Biol 104:47-83.

Guo JU, Su Y, Zhong C, Ming G, Song H. 20II. Hydroxylation of 5-Methylcytosine by TETI promotes active DNA demethylation in the adult brain. Cell 145:423-434.

Hanna and Kelsey, Hanna CW, Kelsey G. 2014. The specification of imprints in mammals. Heredity (Edinb) 113:176-183.

Hendrich B, Hardeland U, Ng HH, Jiricny J, Bird A. 1999. The thymine glycosylase MBD4 can bind to the product of deamination at methylated CpG sites. Nature 401:30I-304.

Hodges $\mathrm{E}$, et al. 20II. Directional DNA methylation changes and complex intermediate states accompany lineage specificity in the adult hematopoietic compartment. Mol Cell 44:17-28

Hogart A, et al. 2012. Genome-wide DNA methylation profiles in hematopoietic stem and progenitor cells reveal overrepresentation of ETS transcription factor binding sites. Genome Res 22:1407-1418.

Hutchins AS, et al. 2002. Gene silencing quantitatively controls the function of a developmental trans-activator. Mol Cell I0:8I-9I.

Jùrgensen HF, Chen Z-F, Merkenschlager M, Fisher AG. 2009. Is REST required for ESC pluripotency? Nature 457:E4-E5.

Joulie M, Miotto B, Defossez P-A. 2010. Mammalian methyl-binding proteins: What might they do. Bioessays 32:1025-1032.

Kaminskas E, et al. 2005. Approval summary: Azacitidine for treatment of myelodysplastic syndrome subtypes. Clin. Cancer Res 11:3604-3608.

Klose RJ, Bird AP. 2006. Genomic DNA methylation: The mark and its mediators. Trends Biochem Sci 31:89-97.

Kohli RM, Zhang Y. 2013. Enzymes, TDG and the dynamics of DNA demethylation. Nature 502:472-479.

Laget S, et al. 2014. MBD4 cooperates with DNMTI to mediate methyl-DNA repression and protects mammalian cells from oxidative stress. Epigenetics 9:546-556.

$\mathrm{Li}$, et al. 2008. A maternal-zygotic effect gene, Zfp57, maintains both maternal and paternal imprints. Dev Cell 15:547-557.

Lienert $\mathrm{F}$, et al. 20I I. Identification of genetic elements that autonomously determine DNA methylation states. Nat Genet 43:1091-1097.

Lim DHK, Maher ER. 2010. Genomic imprinting syndromes and cancer. Adv Genet 70: 145-175

Lister R, et al. 2009. Human DNA methylomes at base resolution show widespread epigenomic differences. Nature 462:315-322.

Liu Y, Toh H, Sasaki H, Zhang X, Cheng X. 2012. An atomic model of Zfp57 recognition of CPG methylation within a specific DNA sequence. Genes Dev 26:2374-2379.

Liu Y, Zhang X, Blumenthal RM, Cheng X. 2013. A common mode of recognition for methylated CpG. Trends Biochem Sci 38:177-183.

Lukic S, Nicolas J-C, Levine AJ. 2014. The diversity of zinc-finger genes on human chromosome 19 provides an evolutionary mechanism for defense against inherited endogenous retroviruses. Cell Death Differ 21:381-387.

Luo S-W, et al. 2009. Regulation of heterochromatin remodelling and myogenin expression during muscle differentiation by FAK interaction with MBD2. EMBO J 28:2568-2582.

Mackay DJG, et al. 2008. Hypomethylation of multiple imprinted loci in individuals with transient neonatal diabetes is associated with mutations in ZFP57. Nat Genet 40:949-95I

Maeda I, et al. 2013. Max is a repressor of germ cell-related gene expression in mouse embryonic stem cells. Nat Commun 4:1754.

Maeder ML, et al. 2013. Targeted DNA demethylation and activation of endogenous genes using programmable TALE-TETI fusion proteins. Nat. Biotechnol 31:| |37-| | 42.

Makar KW, et al. 2003. Active recruitment of DNA methyltransferases regulates interleukin 4 in thymocytes and T cells. Nat Immunol 4:1 I83-1190.

Martinowich K, et al. 2003. DNA methylation-related chromatin remodeling in activitydependent BDNF gene regulation. Science 302:890-893.
Messerschmidt DM, Knowles BB, Solter D. 2014. DNA methylation dynamics during epigenetic reprogramming in the germline and preimplantation embryos. Genes Dev 28:8I $2-828$.

Molaro A, et al. 2014. Two waves of de novo methylation during mouse germ cell development. Genes Dev 28:1544-1549.

Murayama A, et al. 2006. A specific CPG site demethylation in the human interleukin 2 gene promoter is an epigenetic memory. EMBO J 25:1081-1092.

Murgatroyd C, et al. 2009. Dynamic DNA methylation programs persistent adverse effects of early-life stress. Nat Neurosci 12:1559-1566.

$\mathrm{Nitta} \mathrm{H}$, et al. 2013. Three novel ZBTB24 mutations identified in Japanese and Cape Verdean type 2 ICF syndrome patients. J Hum Genet 58:455-460

(transcription factor Gata4 during embryonic stem cell differentiation. PLoS Genet 9:-e 1003574

ikawa $Y$, et al. 2011. The methyl-CpG-binding protein CIBZ suppresses myogenic

differentiation by directly inhibiting myogenin expression. Cell Res 21:1578-1590.

Okano M, Bell DW, Haber DA, Li E. 1999. DNA methyltransferases Dnmt3a and Dnmt3 are essential for de novo methylation and mammalian development. Cell 99:247-257.
Pacaud R, et al. 2014. DNMT3L interacts with transcription factors to target DNMT3L DNMT3B to specific DNA sequences: Role of the DNMT3L/DNMT3B/p65-NFKB complex in the (de-) methylation of TRAFI. Biochimie 104:36-49.

Palacios D, Summerbell D, Rigby PWJ, Boyes J. 2010. Interplay between DNA methylation and transcription factor availability: Implications for developmental activation of the mouse Myogenin gene. Mol Cell Biol 30:3805-38I5.

Palakurthy RK, et al. 2009. Epigenetic silencing of the RASSFIA tumor suppressor gene through HOXB3-mediated induction of DNMT3B expression. Mol Cell 36:219-230.

Petrie K, et al. 2008. Retinoblastoma protein and the leukemia-associated PLZF transcription factor interact to repress target gene promoters. Oncogene 27:5260-5266.

Project Consortium. ENCODE. 2012. An integrated encyclopedia of DNA elements in the human genome. Nature 489:57-74.

Puszyk W, et al. 2013. The epigenetic regulator PLZF represses LI retrotransposition in germ and progenitor cells. EMBO J 32:194I-1952.

Quenneville S, et al. 20II. In embryonic stem cells, ZFP57/KAPI recognize a methylated hexanucleotide to affect chromatin and DNA methylation of imprinting control regions. Mol Cell 44:36I-372

Quenneville S, et al. 20I2. The KRAB-ZFP/KAPI system contributes to the early embryonic establishment of site-specific DNA methylation patterns maintained during development. Cell Rep 2:766-773.

Rowe HM, et al. 2010. KAPI controls endogenous retroviruses in embryonic stem cells. Nature 463:237-240.

Rowe HM, et al. 2013. De novo DNA methylation of endogenous retroviruses is shaped by KRAB-ZFPs/KAPI and ESET. Development |40:519-529.

Serra RW, Fang M, Park SM, Hutchinson L, Green MR. 20I4. A KRAS-directed transcriptional silencing pathway that mediates the $C_{p} G$ island methylator phenotype. Elife 3:-e02313.

Sharif J, et al. 2007. The SRA protein Np95 mediates epigenetic inheritance by recruiting Dnmtl to methylated DNA. Nature 450:908-9/2.

Singh SK, Kagalwala MN, Parker-Thornburg J, Adams H, Majumder S. 2008. REST maintains self-renewal and pluripotency of embryonic stem cells. Nature 453:223-227.

Smith ZD, Meissner A. 2013. DNA methylation: Roles in mammalian development. Nat Rev Genet 14:204-220.

Spruijt CG, et al. 2013. Dynamic readers for 5-(hydroxy) methylcytosine and its oxidized derivatives. Cell I52:1/46-1159.

Stadler MB, et al. 20II. DNA-binding factors shape the mouse methylome at distal regulatory regions. Nature 480:490-495.

Subramaniam D, Thombre R, Dhar A, Anant S. 2014. DNA methyltransferases: A novel target for prevention and therapy. Front Oncol 4:80.

Takikawa S, et al. 2013. Human and mouse ZFP57 proteins are functionally interchangeable in maintaining genomic imprinting at multiple imprinted regions in mouse ES cells. Epigenetics 8:1268-1279.

Tan X, et al. 2013. Zfp8I9, a novel KRAB-zinc finger protein, interacts with KAPI and functions in genomic integrity maintenance of mouse embryonic stem cells. Stem Cell Res II:1045-1059.

Teif VB, et al. 2014. Nucleosome repositioning links DNA (de) methylation and differential CTCF binding during stem cell development. Genome Res 24:1285-1295.

Thillainadesan G, et al. 2012. TGF- $\beta$-dependent active demethylation and expression of the pI5ink4b tumor suppressor are impaired by the ZNF2I7/CoREST complex. Mol Cell 46:636-649.

Thomson JP, et al. 2010. CPG islands influence chromatin structure via the $C_{p}$ G-binding protein Cfpl. Nature 464:1082-1086.

Turelli P, et al. 2014. Interplay of TRIM28 and DNA methylation in controlling human endogenous retroelements. Genome Res 24:1260-1270.

Velasco G, et al. 2010. Dnmt3b recruitment through E2F6 transcriptional repressor mediates germ-line gene silencing in murine somatic tissues. Proc Natl Acad Sci U.S.A 107:9281-9286.

Viré $\mathrm{E}$, et al. 2006. The Polycomb group protein EZH2 directly controls DNA methylation. Nature 439:87|-874.

Wajapeyee N, Malonia SK, Palakurthy RK, Green MR. 2013. Oncogenic RAS directs silencing of tumor suppressor genes through ordered recruitment of transcriptional repressors. Genes Dev 27:2221-2226.

Weaver ICG, et al. 2007. The transcription factor nerve growth factor-inducible protein a mediates epigenetic programming: altering epigenetic marks by immediate-early genes. J Neurosci 27:1756-1768

Weng Y-L, An R, Shin J, Song H, Ming G. 2013. DNA modifications and neurological disorders. Neurotherapeutics 10:556-567.

Wolf D, Goff SP. 2007. TRIM28 mediates primer binding site-targeted silencing of murine leukemia virus in embryonic cells. Cell 131:46-57.

Wolf D, Goff SP. 2009. Embryonic stem cells use ZFP809 to silence retroviral DNAs. Nature 458: $1201-1204$

Wu L, Zheng Q. 2014. Active demethylation of the IL-2 Promoter in CD4+ T cells is mediated by an inducible DNA glycosylase, Myh. Mol Immunol 58:38-49.

Zane L, Sharma V, Misteli T. 2014. Common features of chromatin in aging and cancer: Cause or coincidence. Trends Cell Biol. DOI: 10.1016/j.tcb.2014.07.00I.

Zhang B, et al. 2012. The pl6-specific reactivation and inhibition of cell migration through demethylation of $C_{p} G$ islands by engineered transcription factors. Hum Gene Ther 23:107|-1081

Zhou JC, Blackledge NP, Farcas AM, Klose RJ. 2012. Recognition of CPG island chromatin by Ziller MJ, et al. 2013. Charting a dynamic DNA methylation landscape of the human genome. Nature 500:477-481. 